\section{Malaria Por Plasmodium Falciparum, evolución de un caso}

Plasmodium Falciparum Malaria, evolution of a case

Lissettte Gabriela Armijos Gía

Md.Hospital San Vicente de Paúl,

armijos312@gmail.com,

https://orcid.org/0000-0002-2942-104X

Ingrid Lucely Del Rosario Peralta

Md Centro de Salud tipo C de Caluma,

dra.ingriddelrosario@gmail.com,

https://orcid.org/0000-0003-3401-9976

Gustavo Adolfo Moyón Gusqui

Md Hospital de especialidades Abel

Gilbert Pontón,

drgustavomoyong@gmail.com,

https://orcid.org/0000-0002-6982-3481

Lisbeth Katherine Cevallos Hernández

Md Hospital General del Norte de

Guayaquil Los Ceibos,

drakalisce8@hotmail.com,

https://orcid.org/0000-0001-5121-6360

Anny Nicole Dominguez Vera

Estudiante de medicina de la

universidad de Guayaquil,

annydominguez2215@gmail.com;

Orcid: 0000-0002-0259-8953

Guayaquil - Ecuador

http://www.jah-journal.com/index.php/jah

Journal of American health

Octubre - Diciembre vol. 3. Num. 3 - 2020

Esta obra está bajo una Licencia Creative

Commons

Atribución-NoComercial-CompartirIgual

4.0 Internacional.

RECIBIDO: 18 DE MARZO 2019

ACEPTADO: 28 DE NOVIEMBRE 2019

PUBLICADO: 2 DE JULIO 2020

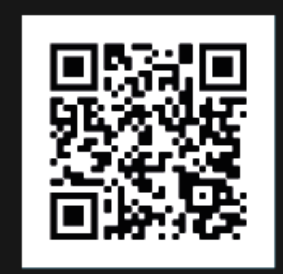

scan this QR

code with your

smart phone or

mobile device to

read more papers

\section{RESUMEN}

La malaria es una enfermedad parasitaria con una amplia distribución en zonas tropicales y subtropicales, endémica en más de 100 países, producida por cuatro especies de plasmodios. Es una patología presente en zonas con abundantes mosquitos Anopheles, por lo que su prevalencia está directamente relacionada con las condiciones socioeconómicas y ambientales de los pacientes. Al tratarse de una enfermedad capaz de producir la muerte rápidamente, es necesario considerarla como una urgencia médica. En este contexto se presenta paciente masculino que acude al servicio de emergencias con un cuadro clínico cuyos síntomas hacen sospechar de un brote de malaria. El diagnóstico oportuno de malaria por Plasmodium falciparum permitió un abordaje óptimo del paciente al cabo de pocos días de realizada la prueba confirmatoria, el examen de gota gruesa y extensión

PALABRAS CLAVE: Malaria, Plasmodium falciparum, antipalúdico, caso

\section{ABSTRACT}

Malaria is a parasitic disease with a wide distribution in tropical and subtropical areas, endemic in more than 100 countries, caused by four species of plasmodia. It is a pathology present in areas with abundant Anopheles mosquitoes, so its prevalence is directly related to the socioeconomic and environmental conditions of the patients. As a disease capable of rapidly causing death, it must be considered a medical emergency. In this context, a male patient case is presented, who comes to the emergency service with a clinical picture which symptoms make us suspect a malaria outbreak. The early diagnosis of malaria by 
Plasmodium falciparum allowed an optimal approach to the patient a few days after the confirmatory test; and after the antimalarial treatment, it allowed a favorable evolution of the patient.
KEYWORDS: fever, mayaro, Chicungunya, Ecuador

\section{INTRODUCCIÓN}

Para la gran mayoría de países que se localizan en regiones tropicales y subtropicales, como Ecuador, la malaria se ha convertido en un problema de salud pública. Se trata de una enfermedad potencialmente mortal causada por parásitos que se transmiten al ser humano por la picadura de mosquitos hembras infectados, del género Anopheles [1].

La malaria es una enfermedad causada por parásitos del género Plasmodium, siendo cuatro las especies que ocasionan la mayoría de las infecciones en los seres humanos: P. vivax; P. falciparum; P. ovale y P. malariae [2]. Se le atribuye especial importancia a la malaria ocasionada por $\mathrm{P}$. falciparum, puesto que su presentación, clínica y evolución es más grave que la infección por cualquiera de los otros agentes causales; y a la malaria causada por P. vivax, con una clínica de similar intensidad y mayor prevalencia en la región de las Américas [3].

Los primeros síntomas comunes (fiebre, dolor de cabeza, escalofríos y vómitos) suelen aparecer 10 a 15 días después de que se haya producido la infección. Son de carácter progresivo y suelen evolucionar sin el tratamiento adecuado. Si no se trata rápidamente con medicamentos eficaces, el paludismo puede ser grave, y a menudo mortal [4]. Por tanto, se hace indispensable el diagnóstico parasitológico preciso y oportuno, especialmente para el futuro manejo que se le debe dar al paciente con ansias de lograr la remisión de su enfermedad [2].

El tratamiento con antipalúdicos es una medida eficaz para curar la enfermedad; sin embargo, es necesario individualizar la especie de Plasmodium causante de la malaria [5]. La OMS también recomienda el uso de artemisina para aquellos casos ocasionados $P$. falciparum; y el uso de cloroquina en casos de P. vivax no resistentes; siendo la terapia combinada con artemisina la piedra angular de este tratamiento.

Los métodos preventivos disponibles actualmente se basan en el control vectorial e incluyen el uso de mosquiteros impregnados de insecticida de larga duración, el rociamiento intradomiciliario con insecticidas, el uso de larvicidas y otras medidas medioambientales para evitar la proliferación de mosquitos en aguas estancadas. [2]. No obstante, aun teniendo estas medidas preventivas, la OMS desde el 2012 recomienda el uso de un tratamiento farmacológico profiláctico (quimioprofilaxis) para aquellas personas que viajan a zonas endémicas [6]

\section{PRESENTACIÓN DEL CASO}

Paciente masculino de 20 años de edad, de nacionalidad ecuatoriana residente actual de la cooperativa Sergio Toral al noroeste de la ciudad de Guayaquil; habita en casa de cemento de dos plantas, cuenta con todos los servicios básicos, frente a un parque descuidado, con maleza y charcos de agua; la vivienda no cuenta con mosquitero, y las ventanas no poseen telas metálicas en buen estado. Sin antecedentes de adicción al alcohol y/o drogas, no refiere alergias, y refiere antecedentes de infección de dengue, no refiere antecedentes familiares. 
Acude a consulta el 29/04/2019 al subcentro de salud y luego a un hospital de especialidades por presentar un cuadro clínico de 5 días de evolución caracterizado por alza térmica no cuantificada, dolor retrocular, cefalea de moderada a intensa holocraneana y dolor abdominal intenso en la región del epigastrio tipo cólico no irradiado no refiere que lo exacerba o que lo calma, acompañado de cuadro diarreico mucosanguinolento fétido en número de 5 en 24 horas. A la exploración física presenta abdomen blando depresible a la palpación en epigastrio, mucosa oral seca, gingivorragia, y sin presencia de edema en las extremidades con signos vitales de TA: 120/70 FC: 84 FR: 20 y So2: $98 \%$ peso: $58 \mathrm{~kg}$ Talla: $1.50 \mathrm{~cm}$. Por lo que realizan examen de gota gruesa para determinar Plasmodium, resultando positivo para Plasmodium falciparum y dengue siendo negativo para IGM e Igg postivo.

Es ingresado con cloruro de sodio IV al 0,9\% $1000 \mathrm{ml} \mathrm{a} 63 \mathrm{ml} / \mathrm{h}$; paracetamol $1 \mathrm{~g}$ ( 2 tabletas de $500 \mathrm{mg}$ ) vía oral; omeprazol $20 \mathrm{mg}$ por vía oral c/6h, primaquina $15 \mathrm{mg}$ VO c/8h, ondasentron y 1 tableta de complejo $b$ al día, donde mostro mejoría de síntomas encontrándose afebril y hermodinámicamente estable.

En la biometría hemática destaca monocitocis de $12.9 \%$ debido a infección aparente, presenta hematíes $\left(4.09 \times 10^{\wedge} 6 / \mathrm{uL}\right)$ que refiere anemia hemolitica; hemoglobina $(11.7 \mathrm{~g} / \mathrm{dl})$; plaquetas (29 x10^3/uL) PDW (8.6) y PCT (0.02\%). En la química sanguínea se observa el TGO 45U/L TGP 46.0U/L fosfatasa alcalina de 103. U/L, LDH $606 \mathrm{U} / \mathrm{L}$. La hemostasia reveló aumento de TP Y TPT y IgG: Positivo para dengue con IgM: negativo. Además, se realizó una ecografía abdominal donde se puede observar:

Hepatomegalia, con contornos regulares de aspecto homogéneo $15.48 \mathrm{~cm}$.

Vesícula poco distendida de paredes finas su contenido naranja.

Esplenomegalia homogénea de $4 \mathrm{~cm}$ por debajo del reborde costal.

Presencia de gas intestinal, sin presencia de líquido libre en la cavidad abdominal.

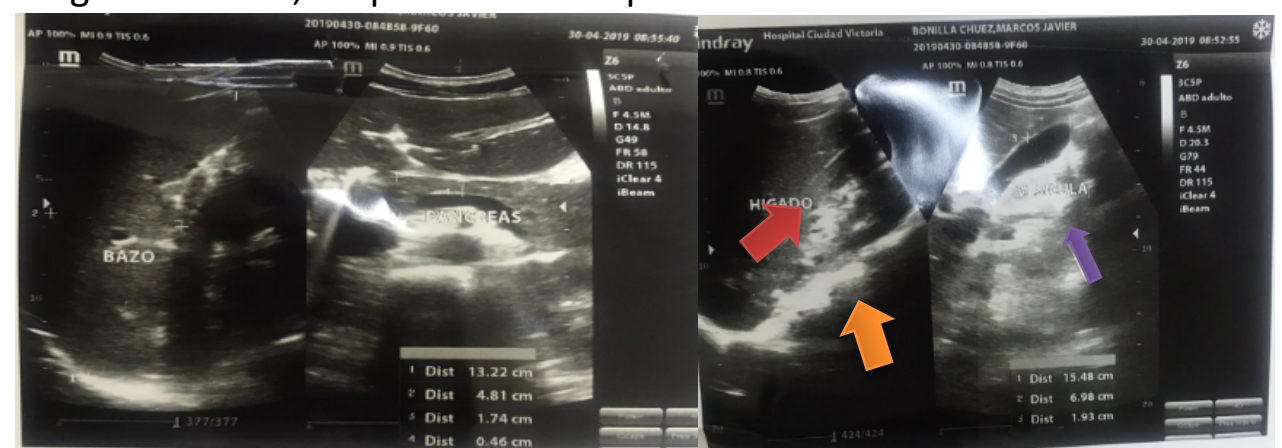

Figura 1: ECOGRAFIA ABDOMINAL

Figura 2: ECOGRAFIA ABDOMINAL

Se le realizo examen de gota gruesa donde resulto positivo para Plasmodium falciparum, El 1 de mayo del 2019, se empezó con el tratamiento antipalúdico (Artemether + Lumefantrina) 4 tabletas a las 8 h00 y 4 tabletas a las 20 h00 por 3 días. Con mejoría notable, persistiendo solo un pico febril que posteriormente en términos de 48 horas remitió todos los síntomas.

\section{DISCUSIÓN}

El paciente presentó una notable mejoría de los síntomas a las 48 horas de iniciado el tratamiento antipalúdico con Artemether + Lumefantrina. Dada la presentación clínica del paciente; la elevación de la temperatura corporal por un periodo de 5 días previos a la consulta, acompañada cefalea intensa con irradiación retroocular, dolor abdominal y diarrea 
mucosanguinloenta, junto con la biometría hemática que reflejaba anemia hemolítica; hacían sospechar de un caso de malaria. Diagnóstico que se confirmó tras el examen de gota gruesa. La Malaria, por lo general, se puede presentar luego de 10 a 15 días tras la infección como un cuadro grave gastrointestinal que cursa con diarrea y vómito que puede ir de leve a moderado, favoreciendo la deshidratación; además de fiebre con escalofríos, malestar general y cefalea intensa, cuadro compatible con el del paciente [7]. La afección hepática es frecuente, por lo que no es raro observar cuadros de ictericia, hepatomegalia y alteraciones moderadas en los marcadores de la función hepática como del aspartato aminotransferasa (AST) y de la alanino amino-tranferasa (ALT); junto con la anemia que puede ser leve o severa, de acuerdo a la evolución del paciente [8]

Bajo estos conceptos, la biometría hemática y la química sanguínea son pruebas que deben considerarse para el seguimiento y control a fin de descartar otras patologías virales que van desde dengue, leptospirosis, fiebre amarilla [2]. La biometría hemática destaca la presencia de anemia, trombocitopenia, y numerosas alteraciones hematológicas y hematopoyéticas, cuya gravedad depende de la especie del parásito que esté implicada, del grado de parasitemia y del estado inmunitario del individuo [9]. En pacientes con malaria por P. falciparum, la anemia oscila del $70 \%$ y al $90 \%$, y entre los mecanismos causantes que se han estudiado, figuran los anticuerpos sobre la membrana del glóbulo rojo, se cree que pueden estar dirigidos contra elementos modificados de la pared del glóbulo rojo o contra proteínas de estadios asexuales del parásito y depositadas sobre la célula durante la rotura al salir el parásito [10]. Sin embargo, es necesario recordar que el diagnóstico definitivo se realiza tras un examen de gota gruesa, el cual debe ser enviado ante la sospecha de malaria, como se realizó con el paciente. Este examen permite determinar la presencia y especia del agente causal, en este caso Plasmodium falciparum, Su determinación es vital para la resolución de los síntomas del paciente.

\section{CONCLUSIONES}

Dada la naturaleza de la enfermedad, y su prevalencia en regiones tropicales y subtropicales en las que no se mantiene un control progresivo del vector, el paludismo ha mostrado una alta incidencia y prevalencia en países subdesarrollados. Hay un vínculo directo entre la calidad de vida y el actual aumento en el número de casos, algo que se ha hecho más evidente en los últimos años con relación a las primeras décadas en las que se propuso el programa de erradicación de la malaria. Por tanto, es vital el correcto diagnóstico, basado en el laboratorio, para el correcto manejo de cada paciente, manejo que debe ser individualizado según el tipo de parásito observado. Por otro lado, en el contexto de la actual pandemia de COVID-19, se puede observar una convivencia entre la pandemia y la transmisión de malaria en los países endémicos, situación que se agrava, no solo en regiones indígenas, sino también en zonas de bajos recursos, donde ya era común observar casos. Según la OPS, este progresivo aumento de casos, desde la actual situación, está vinculado a la atención puesta hacia la pandemia, que desvía los esfuerzos diagnósticos puestos sobre la malaria y a los cambios en las redes de salud. Ante este aumento en el número de casos se vuelve fundamental, entonces, enfocar los esfuerzos en el diagnóstico oportuno que, ante la ausencia de la prevención primaria, es la principal herramienta contra el paludismo.

\section{REFERENCIAS}


1. Ortega Medinal, S. d., Monteagudo Díaz, S., Castro Bruzón, Y., \& Reyes Rodríguez, I. Paludismo por Plasmodium falciparum. Presentación de un caso importado. Medisur [Internet] 2018 [consultado 10 Feb 2020] 16(3), 30-34. Obtenido de Scielo: http://scielo.sld.cu/scielo.php?script=sci_arttext\&pid=S1727-897X2018000300013

2. Campuzano, G; Blair, S. Malaria: consideraciones sobre su diagnóstico. Medicina \& Laboratorio [Internet] 2010 [Consultado 30 Sep 2020] 16(7-8). Disponible en: https://www.medigraphic.com/pdfs/medlab/myl-2010/myl107-8b.pdf

3. Organización Mundial de la Salud. Tratamiento del paludismo: Panorama general. [Internet] 2018. [Consultado 30 Sep 2020] Disponible en: https://www.who.int/malaria/areas/treatment/overview/es/

4. Organización Panamericana de la Salud. Paludismo: Información general [Internet] (s.f.) [Consultado 10 Feb 2020] Obtenido de: https://www.paho.org/hq/index.php?option=com_content\&view=article\&id=2573:20 10-general-information-malaria\&ltemid=2060\&lang=es

5. Tobón A. Malaria: actualización en tratamiento. Redalyc [Internet]. 2009 [Consultado 10 Feb 2020] 29(2). Disponible en: https://www.redalyc.org/pdf/843/84311679016.pdf

6. Carmona J, Álvarez G, Blair S. Malaria por Plasmodium vivax: curación del ataque agudo con tres dosis diferentes de primaquina y dosis fija de cloroquina. Antioquia, Colombia, 20032004. Biomédica [Internet] 2006 [Consultado 30 Sep 2010] 26. Disponible en: http://www.scielo.org.co/img/revistas/bio/v26n3/v26n3a05.pdf

7. Carvajal Guerra V. Malaria. Memorias. [Internet]. Minsalud.gov.co. 2020 [Consultado 10 Feb 2020].

Disponible

en:

https://www.minsalud.gov.co/sites/rid/Lists/BibliotecaDigital/RIDE/VS/TH/memorias_ malaria.pdf

8. Guerrero R, Durán C. Alteraciones hematológicas en pacientes con malaria por Plasmodium vivax en un brote de malaria en Manaure, La Guajira, 2011-2012. Revista Sati [Internet]. 2020 [consultado 10 Feb 2020]; (3):22-23. Disponible en: http://revista.sati.org.ar/index.php/Ml/article/download/348/298

9. Del Mar C. Ictericia y hepatopatía en el paciente con malaria. Infectio [Internet]. 2020 [cited 10 February 2020]. Disponible en: http://www.scielo.org.co/pdf/inf/v14n4/v14n4a06.pdf

10. Malaria | Lab Tests Online-ES [Internet]. Labtestsonline.es. 2018 [consultado 10 Feb 2020]. Disponible en: https://labtestsonline.es/conditions/malaria

11. Organización Panamericana de la Salud. Actualización Epidemiológica: Malaria en las Américas en el contexto de la pandemia de COVID-19 [Internet]. 2020 [Consultado 30 Sep 2020] Disponible en: https://www.paho.org/hq/dmdocuments/2016/2016-chainforme-situacion-malaria-reg.pdf 